\title{
Dynamic Response of RC Cantilever Beam by Equivalent Single Degree of Freedom Method on Elastic Analysis - A Review on Transformation Factors and Dynamic Magnification Factors
}

\author{
Sri Tudjono ${ }^{1, *}$, and Patria Kusumaningrum ${ }^{2}$ \\ ${ }^{1}$ Diponegoro University, Civil Engineering Department, Semarang, Indonesia \\ ${ }^{2}$ ITB, Civil Engineering Department, Bandung, Indonesia
}

\begin{abstract}
The response of multi-degree-of-freedom (MDOF) structure can be correlated to the response of an equivalent single-degree-of-freedom (SDOF) system, implying that the response is controlled by a single, unchanged mode shape. This equivalent SDOF method is eminent as an approximate method of dynamic analysis. In this study, equivalent SDOF method analysis is carried out on RC cantilever beam subjected to dynamic blast loading to review the transformation factors (TFs) provided by TM51300 code.
\end{abstract}

\section{Equivalent SDOF Approach}

This simple approach of equivalent Single Degree of Freedom (SDOF) method to analyze structural elements subjected to blast loads has first been performed by Mays and Smith [1]. A lumped mass of an equivalent SDOF system is proposed to predict the displacement of simplified structures subjected to blast by applying transformation factors of load $\left(K_{L}\right)$, stiffness $\left(K_{S}\right)$, and mass $\left(K_{M}\right)$ to the SDOF analysis. This method follows the TM5-1300 US Army Code [2]. Based on this code, amplitude and time of peak response can be obtained from the idealized charts based on natural period $(T)$ of respective structure.

SDOF method on one way element was studied by Crawford et al. [3] for single column. Big differences between the displacement curves obtained from the numerical analysis and the SDOF analysis were reported. Study on two-way elements was carried out by Morison [4] on walls and slabs. TFs proposed by TM5-1300 for SDOF analyses were reviewed using the yield line theory of plates. Errors were found in some of TFs of two way elements some reaching as much as 50\%. In this study, TFs provided by TM5-1300 code were reviewed on $\mathrm{RC}$ cantilever beam.

\subsection{Elastic Analysis of Cantilever RC Beam}

\footnotetext{
*Corresponding author: tudjono@gmail.com
} 
Multi degree of freedom (MDOF) system can be represented using SDOF system by introducing equivalent dynamic systems. In equivalent dynamic systems, $K_{L}, K_{S}$, and $K_{M}$ are introduced into the system. Triangular blast pressure (Fig. 1a) applied with respect to time is assumed to be uniformly distributed along the height of beam (Fig. 1b). It is then converted into a lateral load (Fig. 1c) applied at the beam's tip after being multiplied by $K_{L}$.

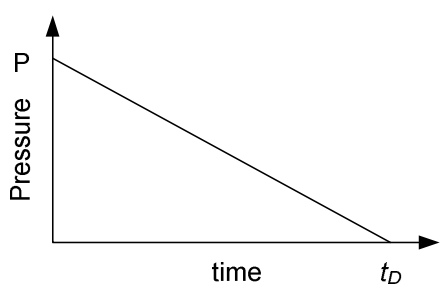

(a)

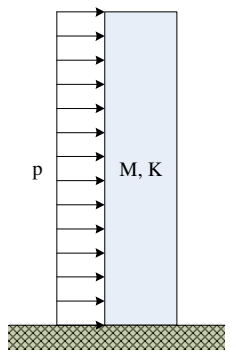

(b)

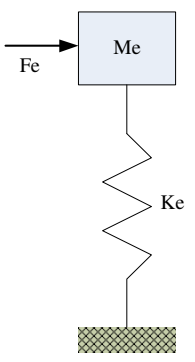

(c)

Fig. 1. (a) Triangular Blast Pressure (b) MDOF System (c) Equivalent SDOF System

The governing equation of the SDOF system may be given in terms of mass $m$, damping $c$, stiffness $k$, force $F$ and displacement $u$ as follows.

$$
m \ddot{u}+c \dot{u}+k u=F(t)
$$

while the governing equation of equivalent SDOF system is given as

$$
m_{e} \ddot{u}+c \dot{u}+k_{e} u=F_{e}(t)
$$

The equivalent terms of mass $m_{e}$, stiffness $k_{e}$ and load $F_{e}$ are as follows

$$
\begin{aligned}
& m_{e}=K_{M} \cdot m \\
& k_{e}=K_{S} \cdot k \\
& F_{e}(t)=K_{L} \cdot F(t)
\end{aligned}
$$

$K_{M}, K_{S}$ and $K_{L}$ refer to the mass, the stiffness and the load transformation factors respectively. The transformation factors, theoretically, are values used to transform the MDOF problems into SDOF system by modeling the deflection as a shape function of $\phi(x)$. The deflection functions of a cantilever RC beam are determined from the boundary conditions. The deflection function assumed for the first mode is represented as

$$
\phi(x)=1-\cos \left(\frac{\pi x}{2 l}\right)
$$

where $x$ is the distance from fixed support whereas $l$ is the beam 's length.

The equivalent mass, stiffness and force are as given in Eq. 5. This equation is equal to Eq. 3. 


$$
\begin{aligned}
& m_{e}=\int_{l} m(x) \phi(x)^{2} d x \\
& k_{e}=\int_{l} E I(x) \phi^{\prime \prime}(x)^{2} d x \\
& F_{e}=\int_{l} q(x) \phi(x) d x
\end{aligned}
$$

Thus, $K_{L}, K_{S}$, and $K_{M}$ are theoretically obtained as :

$$
\begin{gathered}
K_{M}=\frac{\int_{l} \phi(x)^{2} d x}{l}=\frac{\int_{l}\left(1-\cos \left(\frac{\pi x}{2 l}\right)\right)^{2} d x}{l}=\frac{3}{2}-\frac{4}{\pi} \approx 0.227 \\
K_{S}=\frac{\int_{l} \phi^{\prime \prime}(x)^{2} d x}{l^{3}}=\frac{\int_{l}\left(\left(\frac{\pi x}{2 l}\right)^{2} \cos \left(\frac{\pi x}{2 l}\right)\right)^{2} d x}{l^{3}}=\frac{\pi^{4}}{32} \approx 3.044 \\
K_{L}=\frac{\int_{l} \phi(x) d x}{l}=\frac{\int_{l}\left(1-\cos \left(\frac{\pi x}{2 l}\right)\right) d x}{l}=1-\frac{2}{\pi} \approx 0.3634
\end{gathered}
$$

The displacement function is derived using the direct integration method of equivalent SDOF system [5]. It is obtained as a function of the displacement and the velocity at time step $i$ and the applied forces at time step $i$ and $i+1$.

$$
\begin{aligned}
& u_{i+1}=u_{i}\left[e^{-\xi \omega_{e} \Delta t}\left(\frac{\xi \omega_{e}}{\omega_{D e}} \sin \omega_{D e} \Delta t+\cos \omega_{D e} \Delta t\right)\right]+\dot{u}_{i}\left[\frac{e^{-\xi \omega_{e} \Delta t}}{\omega_{D e}} \sin \omega_{D e} \Delta t\right]+ \\
& F_{e i}\left[\frac{1}{\omega_{e} k_{e} \Delta t}\left\{2 \xi-\left(\omega_{e} \Delta t+2 \xi\right) e^{-\xi \omega_{e} \Delta t} \cos \omega_{D e} \Delta t\right\}+\left(\frac{1-\xi\left(\omega_{e} \Delta t+2 \xi\right)}{\omega_{D e} k_{e} \Delta t}\right) e^{-\xi \omega_{e} \Delta t} \sin \omega_{D e} \Delta t\right]+ \\
& +F_{e(i+1)}\left[\frac{1}{\omega_{e} k_{e} \Delta t}\left\{\left(\omega_{e} \Delta t-2 \xi\right)+2 \xi e^{-\xi \omega_{e} \Delta t} \cos \omega_{D} \Delta t\right\}+\left(\frac{2 \xi \xi^{2}-1}{\omega_{D e} k_{e} \Delta t}\right) e^{-\xi \omega_{e} \Delta t} \sin \omega_{D} \Delta t\right]
\end{aligned}
$$

Similarly, by using the same procedures as the displacement equation, the velocity function is derived as follows [5]:

$$
\begin{aligned}
\dot{u}_{i+1}= & -u_{i} e^{-\xi \omega_{e} \Delta t}\left[\frac{\xi^{2} \omega_{e}{ }^{2}+\omega_{D e}{ }^{2}}{\omega_{D e}}\right] \sin \omega_{D e} \Delta t+\dot{u}_{i} e^{-\xi \omega_{e} \Delta t}\left[\cos \omega_{D e} \Delta t-\frac{\xi \omega_{e}}{\omega_{D e}} \sin \omega_{D e} \Delta t\right]+ \\
& F_{e i}\left[-\frac{1}{k_{e} \Delta t}+e^{-\xi \omega_{e} \Delta t}\left\{\frac{1}{k_{e} \Delta t} \cos \omega_{D e} \Delta t-\left(\frac{\omega_{e}{ }^{2}}{\omega_{D e} k_{e}}+\frac{\xi \omega_{e}}{\omega_{D e} k_{e} \Delta t}\right) \sin \omega_{D e} \Delta t\right\}\right]- \\
& F_{e(i+1)} \frac{1}{k_{e} \Delta t}\left[\cos \omega_{D e} \Delta t+\frac{\xi \omega_{e}}{\omega_{D e}} \sin \omega_{D e} \Delta t-1\right]
\end{aligned}
$$

\section{Displacement Analysis of the Cantilever RC Beam Subjected to Blast Load}

In this study, 35 cantilever RC beams of different sizes are investigated (Table 1) and subjected to uniformly distributed pressure varies from $0.01 \mathrm{MPa}$ to $0.05 \mathrm{MPa}$, provided that the beam is still in elastic condition. Loading duration $t_{D}$ is varied from 5 to $15 \mathrm{~ms}$ to obtain higher variation of $t_{D} / T$ ratios. $K_{L}$ of 0.4 based on TM5-1300 is first applied to the model [2]. 
Table 1. Model Descriptions of Cantilever Beams

\begin{tabular}{|c|c|c|c|c|c:c|c|}
\hline$B$ & $H$ & $L$ & $T$ & $B$ & $H$ & $L$ & $T$ \\
\hline$(\mathrm{mm})$ & $(\mathrm{mm})$ & $(\mathrm{m})$ & $(\mathrm{ms})$ & $(\mathrm{mm})$ & $(\mathrm{mm})$ & $(\mathrm{m})$ & $(\mathrm{ms})$ \\
\hline 400 & 400 & 2 & 19.2 & 300 & 400 & 2 & 25.5 \\
\hdashline 250 & 650 & 2 & 30.9 & 400 & 300 & 2 & 19.2 \\
\hdashline 650 & 250 & 2 & 12.3 & 300 & 300 & 2 & 25.5 \\
\hdashline 250 & 500 & 2 & 30.9 & 400 & 1000 & 2 & 19.3 \\
\hline 500 & 250 & 2 & 15.5 & 1000 & 400 & 2 & 8.7 \\
\hline 250 & 400 & 2 & 30.9 & 400 & 800 & 2 & 19.3 \\
\hline 400 & 250 & 2 & 19.1 & 800 & 400 & 2 & 10.3 \\
\hline 250 & 350 & 2 & 30.8 & 400 & 600 & 2 & 19.3 \\
\hline 350 & 250 & 2 & 19.1 & 600 & 400 & 2 & 13.2 \\
\hline 250 & 300 & 2 & 30.8 & 400 & 500 & 2 & 19.2 \\
\hline 300 & 250 & 2 & 25.4 & 500 & 400 & 2 & 15.6 \\
\hline 250 & 250 & 2 & 30.8 & 500 & 500 & 2 & 15.6 \\
\hline 300 & 800 & 2 & 25.6 & 600 & 600 & 2 & 13.3 \\
\hline 800 & 300 & 2 & 10.3 & 600 & 500 & 2 & 13.2 \\
\hline 300 & 600 & 2 & 25.6 & 500 & 600 & 2 & 15.6 \\
\hline 600 & 300 & 2 & 13.2 & 250 & 600 & 2 & 30.9 \\
\hline 300 & 500 & 2 & 25.6 & 600 & 250 & 2 & 13.1 \\
\hline 500 & 300 & 2 & 13.2 & & & \\
\hdashline
\end{tabular}

It is shown in Fig. 2 that for the case of a cantilever RC beam subjected to blast load over a range of applied pressures, the peak displacement which occurs at the tip of the beam plotted against $t_{D} / T$ follows a power function. The results also show that under elastic condition, for structures with the same $t_{D} / T$ ratio, a $5 \mathrm{~ms}$ longer of loading duration $t_{D}$, results in higher peak displacement at the cantilever tip of up to 5 times.

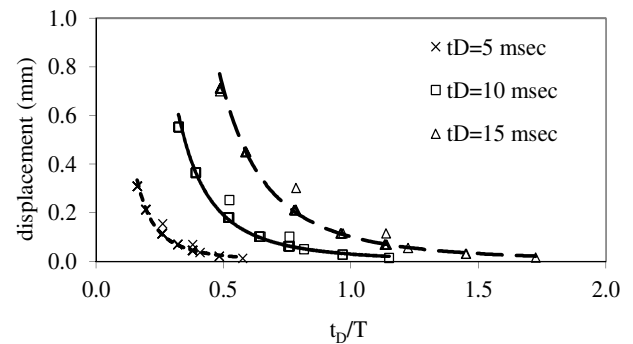

(a)

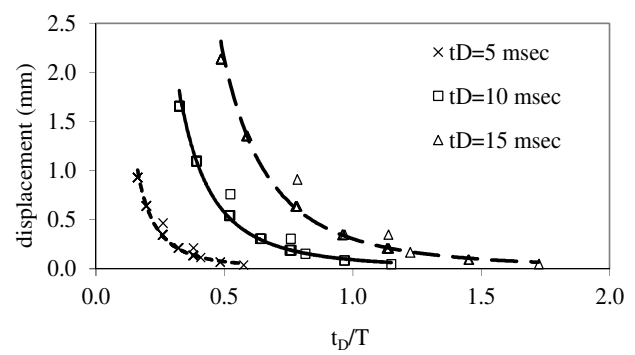

(b) 


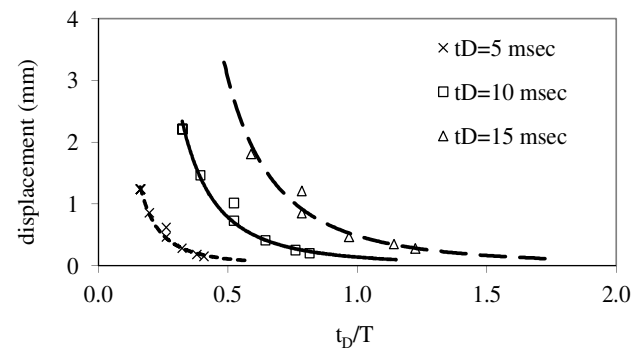

(c)

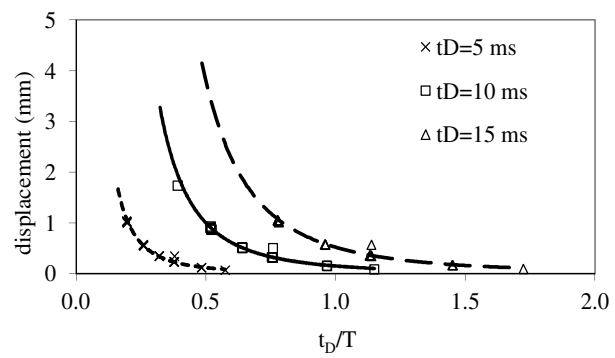

(d)

Fig. 2. Peak displacements of beams subjected to blast pressures of (a) $0.01 \mathrm{MPa}$, (b) 0.03 $\mathrm{MPa}$, (c) $0.04 \mathrm{MPa}$ and (d) $0.05 \mathrm{MPa}$

\subsection{Dynamic Magnification Factor (DMF)}

DMF is the ratio of the displacement under dynamic loading $F\left(F_{0}, t\right)$ to the displacement under the static loading $F_{0}$. The DMF value of a beam differs in time following trigonometric function. DMF equation is derived [5] and given, based on its intervals of time and loading duration $t_{D}$ as follows :

$$
\begin{gathered}
D M F=\frac{u}{u_{\text {static }}}=1-\cos \omega_{e} t+\frac{1}{\omega_{e} t_{D}} \sin \omega_{e} t-\frac{t}{t_{D}}, \text { for } 0 \leq t \leq t_{D} \\
D M F=\frac{u}{u_{\text {static }}}=\frac{1}{\omega_{e} t_{D}}\left\{\sin \omega_{e} t-\sin \omega_{e}\left(t-t_{D}\right)\right\}-\cos \omega_{e} t, \text { for } t \geq t_{D}
\end{gathered}
$$

As shown in Fig. 3, the maximum value of DMF depends upon the ratio of $t_{D} / T$. The higher the ratio of $t_{D} / T$, the DMF obtained is greater. Once the ratio of $t_{D} / T$ reaches a value larger than 1, the slope of the curve reduces until the DMF reaches the maximum value of 2 . The DMF analysis can therefore be used as to validate the results obtained from the theoretical equivalent SDOF analysis. Fig. 3 also shows a comparison between the DMF value obtained from the structural dynamic theory and from SDOF analysis. It is observed that the DMF of SDOF results are in good agreement with the theoretical values.

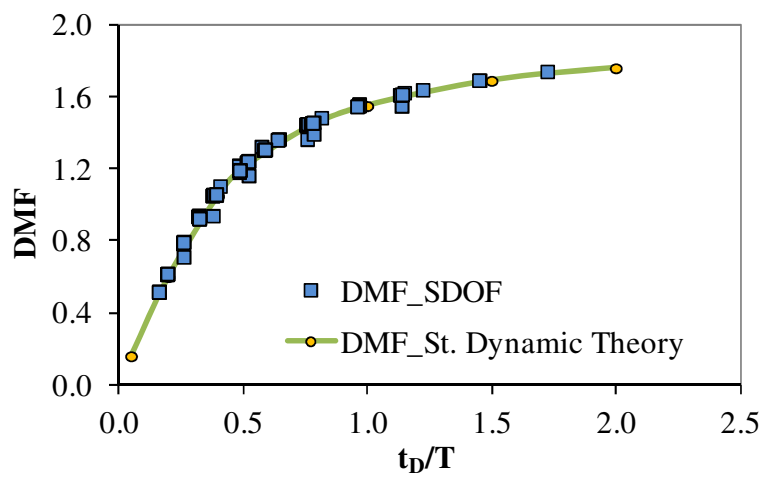

Fig. 3. Maximum DMF 


\subsection{Transformation Factor (TF)}

On the basis of load transformation factor $\left(K_{L}\right)$, TM5-1300 proposed a value of 0.40 . It slightly overestimates the $K_{L}$ value derived by the structural dynamics theory which provides a unique $\mathrm{KL}$ value equal to 0.363 (Fig. 4). With regards to the mass transformation factors $\left(K_{M} \mathrm{~s}\right)$, TM5-1300 proposed a $K_{M}$ value of 0.26 , again, higher than $K_{M}$ derived using structural dynamics theory which is given as 0.227 .

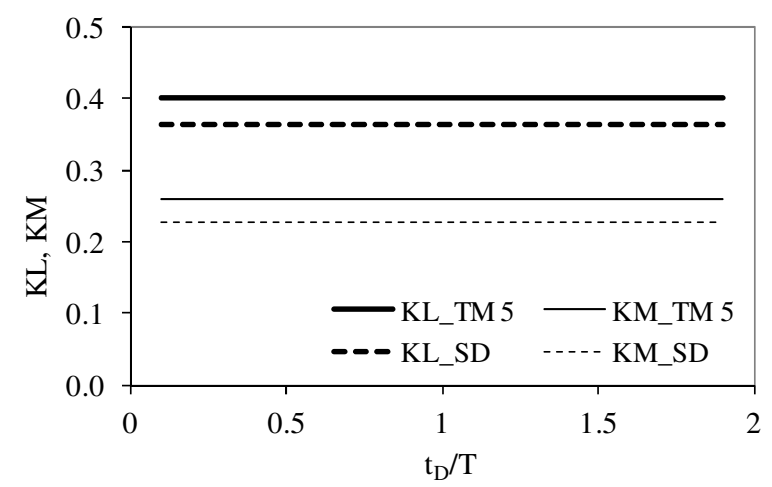

Fig. 4. Transformation Factors

\section{Conclusion}

With respect to DMF, the DMFs obtained from the SDOF analysis are found to be in good agreement with those obtained from the structural dynamics theory. On load and mass transformation factors, TM5-1300 provides an upper bound value, meanwhile the structural dynamics theory gives a lower bound value. The TM5-1300 may use different approach with respect to deflection function and may include additional safety factor of 10 to $15 \%$ for the purpose of blast resistant design.

\section{References}

1. C. G. Mays, P. D. Smith, Blast effects on buildings (Thomas Telford Publication, London, 1995)

2. TM5-1300, Structures to resist the effects of the accidental explosions (US Department of Army, New Jersey, 1990)

3. J. E. Crawford, J. L. Malvar, J. W. Wesevich, J. Valancius, D. R. Aaron, Retrofit of RC structures to resist blast effects. ACI Struct J, 94, 371-377 (1997)

4. C. M. Morison, Dynamic response of walls and slabs by single-degree-of-freedom analysis-a critical review and revision, Int J Impact Eng, 32, 1214-1247 (2006)

5. P. Kusumaningrum, Numerical Modeling of RC and ECC Encased RC Columns Subjected to Close-in Explosion (PhD Thesis, NUS, Singapore, 2010) 